\title{
LAS JORNADAS CIENTÍFICAS FORTALECEN LA ENSEÑANZA- APRENDIZAJE DE ASPECTOS BIOLÓGICOS EN EDUCACIÓN PRIMARIA Y SECUNDARIA
}

\section{The Scientific Days as Tools that Strengthen the Teaching-learning of Biological Aspects in Primary and Secondary Education}

Miguel Guevara*

Instituto Superior de Formación Docente Salomé Ureña,

República Dominicana

\} \text { miguel.guevara@isfodosu.edu.do }

ID https://orcid.org/0000-0002-1830-3822

\section{Mairín Lemus-Barrios}

Instituto Superior de Formación Docente Salomé Ureña, República Dominicana

■mairin.lemus@isfodosu.edu.do

ID https://orcid.org/0000-0002-1657-0505

\section{Resumen}

Este artículo de revisión aborda las Jornadas Científicas escolares como herramientas que fortalecen los procesos de enseñanza-aprendizaje en el área de Biología, en estudiantes de educación primaria y secundaria. La revisión bibliográfica ha puesto de manifiesto que las actividades prácticas en los laboratorios o en jornadas científicas escolares juegan un papel preponderante en la enseñanza de las disciplinas científicas, con importantes beneficios e incremento del aprendizaje en los alumnos, lo cual permitiría de forma directa afianzar los conocimientos biológicos en los estudiantes de secundaria y disminuir así las dificultades que suelen tener con este tipo de asignatura al ingresar a la educación superior, al mismo tiempo, despertar el interés por el estudio de las ciencias.

Palabras clave: biología, jornadas científicas, conocimiento científico escolar, método de enseñanza, proceso de enseñanza, estrategia de enseñanza, técnica de enseñanza, método educativo.

\begin{abstract}
This review deals with the subject related to the scientific school days as tools that strengthen the teaching-learning of biological aspects in students of primary and secondary education. The literature review has shown that practical activities in laboratories or school science days play a leading role in the teaching of scientific disciplines, with important benefits and increased learning in students, which would directly strengthen the biological knowledge in secondary students and thus reduce the difficulties that usually have with this type of subject to enter higher education and at the same time arouse interest in the study of science.

Keywords: biology, teaching methods, school science knowledge, teaching techniques, instructional methods, educational methods, teaching strategies.
\end{abstract}

ISSN (impreso): 2636-2139

ISSN (en línea): 2636-2147

Sitio web: https://revistas.isfodosu.edu.do/recie
* Autor de correspondencia

Recibido: 01 octubre 2018

Aprobado: 14 noviembre 2018

\section{COMO CITAR:}

Guevara, M., \& Lemus-Barrios, M. (2019). Las jornadas científicas fortalecen la enseñanza-aprendizaje de aspectos biológicos en educación primaria y secundaria. Revista Caribeña de Investigación Educativa (RECIE), 3(1), 95-101. https://doi.org/10.32541/recie.2019.v3i1.pp95-101 


\section{Introducción}

Muchos de los fenómenos físicos, químicos y biológicos que son enseñados en la escuela primaria y secundaria, a través de conocimientos teóricos, difícilmente acercan a los estudiantes a la comprensión de dichos eventos; en muchos casos, por la difícil asociación entre la teoría y la práctica, o entre lo conceptual y lo real.

El proceso de aprendizaje de las áreas científicas, particularmente la Biología, donde los aspectos conceptuales son acompañados de experimentos sencillos o interacción con los ecosistemas, puede despertar el interés en los estudiantes para comprender, vivir y experimentar el conocimiento de las ciencias de una forma divertida, agradable y apasionante.

En este sentido, existen muchas iniciativas gubernamentales que han fomentado el interés por las ciencias, como la reunión de la CILAC 2016 (Foro Abierto de Ciencias, Latinoamérica y el Caribe), donde se señala en uno de sus planteamientos que la educación científica debe asegurar a todos sus estudiantes aprendizajes de calidad. Sin embargo, el escenario de la región muestra claramente que, en estos niveles del sistema educativo, la educación no solo no brinda estos aprendizajes, sino que la manera como se presenta el conocimiento científico tiende a que los jóvenes pierdan el interés por aprender ciencias, y no se despierten vocaciones científicas (Macedo, 2016)

Así mismo, en la Conferencia Mundial sobre la Ciencia para el siglo XXI, auspiciada por la UNESCO y el Consejo Internacional para la Ciencia, se declaraba:

Para que un país esté en condiciones de atender a las necesidades fundamentales de su población, la enseñanza de las ciencias y la tecnología es un imperativo estratégico. Como parte de esa educación científica y tecnológica, los estudiantes deberían aprender a resolver problemas concretos y a atender a las necesidades de la sociedad, utilizando sus competencias y conocimientos científicos y tecnológicos (Declaración de Budapest, 1999).

De acuerdo con algunos estudios, para que las nuevas generaciones se interesen por las ciencias deben entrar en contacto con contenidos científicos y desarrollar algún gusto por el área antes de alcanzar los 15 años de edad. Si esto no ocurre, cuando lleguen a adultos no se interesarán por la ciencia o no serán capaces de crear y manejar conocimientos científicos y tecnológicos (Frascati Manual, 2002).

La Prueba de Aprendizaje de Ciencias y Matemáticas en la educación secundaria que se aplica a través del programa PISA, creado por la Organización para la Cooperación y el Desarrollo Económico (OCDE), está dirigida a jóvenes de 15 años miembros de la OCDE (método de mayor reconocimiento para la evaluación de resultados del aprendizaje en la Educación Secundaria). Dicha prueba ha mostrado que los jóvenes latinoamericanos de 15 años carecen de destrezas básicas en materia de Aritmética, así como de nociones elementales de conocimientos científicos. Entre los países evaluados, Colombia, Brasil y Argentina mostraron el desempeńo más bajo con relación a Chile, Uruguay y México (Banco Interamericano de Desarrollo, 2010)

Existen diversas formas de atraer el interés hacia las ciencias en los estudiantes de primaria y secundaria, una de ellas son las jornadas científicas, que si bien es cierto no son aplicadas ampliamente a nivel mundial, sí han existido numerosas iniciativas. Las jornadas científicas representan para los estudiantes de primaria y secundaria lo que para los científicos los congresos. A este nivel, los estu- 
diantes tendrán la oportunidad de conocer de cerca qué es un experimento, cómo se lleva a cabo y qué aprenden en sus actividades.

\section{Revisión de la literatura}

En la actualidad, en diferentes instituciones de educación superior en República Dominicana, se han observado estudiantes de nuevo ingreso a carreras agrobiológicas y de la salud, con escasos conocimientos básicos en aspectos biológicos, tales como: manejo de microscopios, estereoscopios, pHmetros y termómetros, entre otros. Así mismo, muestran incoherencias en la formulación de preguntas de investigación, análisis de los resultados y conclusiones de experimentos sencillos que permiten tener una visión amplia de un determinado proceso biológico.

La situación antes planteada ha surgido debido a que, en los centros de Educación Primaria y Secundaria, la Biología, así como otras disciplinas científicas, se enseña, principalmente, mediante aspectos $100 \%$ teóricos, lo cual disminuye la capacidad de aprendizaje y la motivación de los estudiantes.

Ante esta situación, es imperativa la implementación de actividades científicas escolares, entre ellas las jornadas científicas, que no son algo nuevo, y aunque su aplicación actual no sea tan notoria, se ha demostrado su valor como una valiosa herramienta para la adquisición de conocimientos (Retana Alvarado, Vázquez Bernal \& Camacho Álvarez, 2018), y además, su ejecución no requiere grandes inversiones ni contar con personal altamente especializado.

Las actividades en los laboratorios, a juicio de Lightburn y Fraser (2007), tienen un papel fundamental para el aprendizaje de los alumnos en la enseńanza de las disciplinas científicas. En efecto, esas actividades son muy importantes porque les dan a los estudiantes la oportunidad de conseguir destrezas y habilidades al realizar las prácticas y al ser la dinámica del laboratorio muy diferente a la de una clase teórica.

De manera similar, las investigaciones de Séré (2002) destacan que la actividad experimental aporta más a la enseńanza que otros métodos utilizados para tal fin. Esta autora muestra que el trabajo práctico facilita la comprensión y que el aprendizaje conceptual ayuda a hacer ciencia, a la vez que favorece la motivación de los estudiantes, así como su iniciativa y autonomía. También indica que tales actividades prácticas posibilitan la construcción de modelos que permitan que la teoría pueda ser asimilada de una manera más efectiva, mostrando así que la experimentación favorece la adquisición de conceptos.

En ese mismo orden de ideas, Krasilchik (2004) acentúa el valor de las actividades experimentales en la enseñanza de Biología, pues permiten que los alumnos tengan contacto directo con los fenómenos, manipulando materiales y equipamiento, y observando organismos. Además, refuerza que el contacto de los alumnos con resultados no previstos desafíe su imaginación y razonamiento, permitiendo que convivan con distintas etapas, como la manipulación, la observación, la investigación y la interpretación.

Martínez-Illescas (2015), por su parte, expuso que para aprender y comprender los hechos o sucesos que ocurren en nuestro entorno, los estudiantes desde edades tempranas y a través de la experimentación deben relacionar los conocimientos de ciencia, tecnología y sociedad, para enfrentarse a los retos o problemas cotidianos del medio que les rodea.

Con relación a la efectividad de las jornadas científicas escolares, incluidas las exposiciones cien- 
tíficas, experimentos guiados y diseño de murales, entre otros; Oliva et al. (2004) expresan que este tipo de actividad presenta una serie de ventajas con respecto a otras estrategias educativas, entre las que destacan: 1) Implicación directa del alumno en el proceso de enseńanza-aprendizaje, fomentando actitudes positivas hacia la ciencia. 2) Creación de una actitud de colaboración, participación y responsabilidad en el alumnado. 3) Proporciona un entorno en el que se desarrolla favorablemente el clima de relaciones humanas. 4) Transfiere el protagonismo al estudiante, lo cual mejora su autoestima a través de una experiencia repleta de obstáculos y dificultades, pero que al final pudiera aportar la satisfacción de ver reconocido su trabajo. 5) Posibilita la transferencia de las habilidades adquiridas al aprendizaje formal de las aulas.

De acuerdo con Bencze y Bowen (2009), las ferias de ciencias facilitan el desarrollo de habilidades, concepciones sobre los fenómenos científicos y confianza para la resolución de problemas, influyendo no sólo en los conocimientos, sino en la autonomía, y pasan a contribuir en el proceso de enseñanza-aprendizaje. Por su parte, Fernández (2013) evidenció que los trabajos prácticos de laboratorio promueven en los estudiantes la construcción de modelos conceptuales en la enseńanza de la Biología y ofrecen una visión correcta de la actividad científica.

Sobre este particular, la presente revisión pone de manifiesto cómo las jornadas científicas en instituciones de Educación Secundaria coadyuvan activamente al aprendizaje de las ciencias biológicas, así como de otras disciplinas de las ciencias naturales, por lo que al brindar la oportunidad de participar en experimentos científicos entretenidos y sencillos, los educandos fijan mejor los contenidos y se evita la memorización.

\section{Experiencias}

En Costa Rica, Arce (2002) evidencia que, en general, a los niños de edad escolar les fascina la realización de experimentos científicos en sus clases de ciencias. El autor, después de analizar las encuestas aplicadas, tanto a estudiantes como a profesores, concluye que:

- La mayoría de los maestros y maestras están conscientes de la conveniencia de apoyar los temas científicos con algún tipo de experimento, aunque reconocen que les falta mayor capacitación en este campo.

- Es falsa creencia pensar que para realizar experimentos se requiere de una gran inversión de tiempo y dinero.

- Hoy, más que antes, se hace indispensable una revisión de los métodos que se están utilizando en la enseñanza de las ciencias naturales en nuestras escuelas.

- Es necesario un cambio de actitud en el docente en cuanto a la manera de enfrentarse con el conocimiento científico y su utilidad para comprender el mundo en que vivimos. Ya no se puede concebir la enseñanza de la ciencia de una forma memorística y sin razonamiento.

En España, Oliva, Matos y Acevedo (2008) demostraron que las exposiciones científicas escolares motivan a los estudiantes a desarrollar otros factores asociados a la dimensión afectiva del aprendizaje de las ciencias y contribuyen en el desarrollo profesional de los profesores de Educación Secundaria, quienes intercambian experiencias con otros colegas y adquieren conocimientos didácticos al participar de procesos de formación continua y elaboración de módulos de exposición con sus estudiantes. 
Silveira (2014) en su investigación, realizada en Uruguay, manifestó que los niños desarrollan el pensamiento científico en la medida en que resuelven diferentes situaciones o problemas. De igual forma, el autor expresó que las valoraciones de los participantes, tanto de los talleres regulares como de los especiales, muestran un grado de satisfacción muy alto, encontrando una aplicación de los conceptos y destrezas adquiridas a las actividades de aula y a la vida cotidiana.

En un estudio exploratorio, Retana Alvarado y Vázquez Bernal (2016) distinguen los componentes del proceso de ferias de ciencia y tecnología de Costa Rica, que motivan en el estudiantado de Bachillerato la elección de carreras científicas y tecnológicas de la Educación Superior. Los resultados de esta investigación sugieren que existen componentes motivantes de las ferias, tales como el desarrollo de competencias científicas, el fomento de actitudes favorables, el acercamiento al quehacer científico, que influyen en la selección de carreras en esas áreas.

El artículo de Cuevas, Hernández, Leal y Mendoza (2016) muestra que luego de aplicar un cuestionario estandarizado a 1,559 estudiantes y una entrevista a 74 docentes y 35 directores de 35 escuelas primarias públicas y particulares en 14 ciudades del país, los estudiantes que participaron en el estudio adoptan una actitud positiva hacia la ciencia y una opinión favorable hacia materias como Ciencias Naturales y Matemáticas.

Existen iniciativas por parte de los gobiernos para mejorar significativamente los conocimientos prácticos de las ciencias en las instituciones escolares, tomando en consideración desde los más pequeńos hasta los adolescentes. A criterio de los autores mencionados, esta actitud positiva debe ser aprovechada para la enseńanza y el aprendizaje de la ciencia y la tecnología, así como para la continua formación de la vocación científica y de posibles futuros investigadores, ya que esta dimensión actitudinal otorga a las ciencias y a la investigación un mayor interés, motivación, facilidad de aprendizaje, relevancia social y personal; utilidad para la vida al comprender su entorno y generar soluciones a problemas actuales; por último, posibilita una mayor comprensión de la ciencia y la tecnología, lo que contribuye significativamente a la alfabetización científica de todas las personas.

Otras investigaciones que destacan la importancia de las jornadas escolares de ciencia o las actividades experimentales como herramienta didáctica para el aprendizaje de la Biología (Cuevas et al., 2016; García Ruiz, 2001).

En República Dominicana, las jornadas científicas escolares, como estrategias metodológicas en el aprendizaje de diferentes áreas de la Biología, a nivel de estudios de primaria y secundaria, no han sido referidas en publicaciones científicas, sin embargo, sí se han realizado reseñas periodísticas de diferentes eventos que tienen que ver con esta materia. Entre estas reseñas, se incluyen:

- https://listindiario.com/elnorte/2018/04/27/512426/escuela-celebra-feria-cientifica-y-tecnologica

- https://listindiario.com/la-vida/2010/4/26/139827/Celebran-la-Feria-Cientifica-Distrital

\section{Consideraciones finales}

El análisis de la bibliografía consultada evidenció que la implementación de talleres, ferias o jornadas científicas, en recintos de Educación Primaria y Secundaria, pueden fortalecer la enseñanza-aprendizaje de aspectos vinculados con la Biología, lo cual permitiría el éxito académico de los 
estudiantes en las carreras universitarias afines. Todo esto es posible debido a que estos talleres de ciencias promueven el desarrollo de capacidades, conocimientos y técnicas, a través de una invitación al descubrimiento, que aumentan el interés de los participantes por saber, cada vez más, sobre los diversos campos del área y sus posibilidades de aplicación en la tecnología (Scassa, 2015).

Conociendo el amplio trabajo que despliegan los organismos internacionales para fortalecer el estudio de las ciencias en los países de Latinoamérica, es importante que se fomenten actividades de este tipo, a fin de avanzar en la cultura de las ciencias, particularmente en República Dominicana, donde no es mucho lo que se ha hecho en este sentido.

Finalmente, derivada de los planteamientos anteriores, partimos de la siguiente hipótesis: los estudiantes de educación secundaria, al participar en las ferias de ciencia y tecnología, experimentan una práctica positiva que los motiva en el desarrollo de su vocación científica y que incide en las decisiones de preferencia y elección de carreras relacionadas cuando ingresan a la universidad. Estos planteamientos están en concordancia con los expuestos por Fouad (2007), quien expresa que la elección vocacional se construye a partir del conocimiento que cada persona tiene de sí misma y de la profesión, así como de la influencia del contexto social sobre el individuo, y por Vázquez-Alonso, Manassero-Mas y Taberner-Ferrer (2012), quienes aprecian que entre las experiencias escolares que motivan más a los alumnos en la elección de una carrera de ciencia y tecnología se encuentran las ferias científicas.

\section{Agradecimientos}

Esta investigación fue financiada parcialmente a través del proyecto PI-018-2018, del Instituto Superior de Formación Docente Salomé Ureña (ISFODOSU), República Dominicana.

\section{Referencias}

Arce, M. (2002). El valor de la experimentación en la enseñanza de las Ciencias Naturales. El taller de ciencias para niños de la Sede del Atlántico de la Universidad de Costa Rica: Una experiencia para compartir. Revista Educación, 26(001), 147-154.

Banco Interamericano de Desarrollo. (2010). Ciencia, tecnología e innovación en América Latina y el Caribe. Un compendio estadistico de indicadores. Washington, USA: BID.

Bencze, J. L., \& Bowen, G. M. (2009). A national science fair: Exhibiting support for the knowledge economy. International Journal of Science Education, 31(8), 2459-2483. https://doi.org/10.1080/09500690802398127

Cuevas, A., Hernández, R., Leal, B. E., \& Mendoza, C. P. (2016). Enseñanza-aprendizaje de ciencia e investigación en educación básica en México. Revista Electrónica de Investigación Educativa, 18(3), 187-200.

Declaración de Budapest. (1999). Marco general de acción de la Declaración de Budapest. Recuperado de http://bit.ly/2W2NiWg

Fernández, N. (2013). Los trabajos prácticos de laboratorio por investigación en la enseñanza de la Biología. Revista de Educación en Biología, 16(2), 15-30.

Fouad, N. A. (2007). Work and vocational psychology: Theory, research, and applications. Annual. Review of Psychology, 58, 543-564. https://doi.org/10.1146/annurev.psych.58.110405.085713 
Frascati Manual (2002). Proposed standard practice for surveys on research and experimental development. Paris: OECD.

García Ruiz, M. (2001). Las actividades experimentales en la escuela secundaria. Perfles educativos, 23(94), 70-90.

Krasilchik, M. (2004). Prática de Ensino de Biología. Sáo Paulo: Universidade de São Paulo.

Lightburn, M. E., \& Fraser, B. J. (2007). Classroom environment and student outcomes among students using anthropometry activities in high-school science. Research in Science \& Technological Education, 25(2), 153-166. https://doi.org/10.1080/02635140701250576

Macedo, B. (2016). Educación científica. Montevideo, Uruguay: UNESCO.

Martínez-Illescas, M. (2015). La importancia de los experimentos pautados en Educación Primaria (Tesis doctoral). Universidad de Valladolid, Valladolid.

Oliva, J. M., Matos, J., Bueno, E., Bonat, M., Domínguez, J., Vázquez, A., \& Acevedo, J. A. (2004). Las exposiciones científicas escolares y su contribución en el ámbito afectivo de los alumnos participantes. Enseñanza de las Ciencias: Revista de Investigación y Experiencias Didácticas, 22(3), 425-439.

Oliva, J. M., Matos, J., \& Acevedo, J. A. (2008). Contribución de las exposiciones científicas escolares al desarrollo profesional docente de los profesores participantes. Revista Electrónica de Enseñanza de las Ciencias, 7(1), 178-198. Recuperado de http://bit.ly/2Q3U9cG

Retana Alvarado, D. A., \& Vázquez Bernal, B. (2016). Influencia de las ferias de ciencia y tecnología de Costa Rica en la elección de estudios superiores científicos y tecnológicos. En M. Cañada (Ed.), Ciencias para comprender el mundo. Investigación e innovación en Didáctica de las Ciencias Experimentales (pp. 279-295). Madrid: ENTINEMA.

Retana Alvarado, D. A., Vazquéz Bernal, B., \& Camacho Álvarez, M. M. (2018). Las ferias de ciencia y tecnología de Costa Rica y sus aportes a la Educación Secundaria. Revista Electrónica Actualidades Investigativas en Educación, 18(2), 1-43. Recuperado de https://bit.ly/2w1s6kX

Scassa, A. M. (2015). El Taller de Ciencias como semillero de futuros científicos. Recuperado de http://bit.ly/2vT26bs

Séré, M. G. (2002). La enseñanza en el laboratorio: ¿Qué podemos aprender en términos de conocimiento práctico y de actitudes hacia la ciencia? Enseñanza de las Ciencias, 20(3), 357-368.

Silveira, F. (2014). Los talleres de ciencia como herramienta para el fomento de la cultura cientifica. Trabajo presentado en el Congreso Iberoamericano de Ciencia, Tecnología, Innovación y Educación. Buenos Aires, Argentina. Recuperado de http://bit.ly/2HiJGWQ

Vázquez-Alonso, Á., Manassero-Mas, M. A., \& Taberner-Ferrer, J. (2012). Factores que influyen sobre la elección de estudios superiores de ciencias y tecnología. Trabajo presentado en VII Seminario Ibérico/III Seminario Iberoamericano CTS en la Enseñanza de las Ciencias, Madrid, España. 